\title{
Níveis de inclusão do bagaço de mandioca na ração de frangos de crescimento lento: características físico-químicas da carne ${ }^{1}$
}

\section{Levels of inclusion in cassava bagasse chicken feed of slow growth: physical and chemical characteristics of meat}

\author{
Aline Ferreira Amorim²*; Jefferson Costa de Siqueira ${ }^{3}$; Kênia Ferreira Rodrigues ${ }^{4}$; \\ Roberta Gomes Marçal Vieira Vaz ${ }^{4}$; Silvia Minharro Barbosa ${ }^{4}$; \\ Helcileia Dias Santos ${ }^{4}$; Fabiana Cordeiro Rosa ${ }^{4}$; Joana Patrícia Lira de Sousa ${ }^{5}$; \\ Elisson Gomes da Silva ${ }^{6}$; Isabela Maria Machado de Oliveira Moufarreg ${ }^{7}$; \\ Iberê Pereira Parente ${ }^{8}$; José Ailton Rodrigues Soares ${ }^{9}$
}

\section{Resumo}

O objetivo desse estudo foi avaliar o efeito dos níveis de inclusão $(0,10,20$ e 30\%) do bagaço de mandioca $(\mathrm{BM})$ nas rações sobre os parâmetros físicos e químicos da carne crua e cozida da coxa, sobrecoxa e peito de frangos de corte tipo caipira da linhagem Label rouge $\mathbb{R}$, abatidos aos 84 dias de idade. As análises foram realizadas no laboratório de nutrição animal da Universidade Federal do Tocantins - UFT, Escola de Medicina Veterinária e Zootecnia, campus de Araguaína. Quarenta carcaças foram divididas em meias-carcaças, sendo 10 meias-carcaças para análise da composição química da carne crua e as outras 10 meias-carcaças para análise da composição química da carne cozida. Nessas análises estão incluídos os teores de proteína bruta (PB), extrato etéreo (EE), matéria seca (MS) e cinzas (CZ), com base na percentagem de matéria seca, além da energia bruta (EB) e das perdas por cozimento. Também realizou-se análises dos atributos físicos da carne (cor, pH e textura) nas outras 20 meiascarcaças. A inclusão do BM na dieta de frangos de corte tipo caipira e a cocção afetaram $(\mathrm{P}<0,05)$ as características químicas da carne. Observou-se na carne da coxa redução linear na deposição de CZ e aumento linear na deposição de PB. Na carne da sobrecoxa o teor de PB aumentou até o nível estimado de $17,22 \%$ de inclusão de BM correspondente à deposição de $84,21 \%$ de PB; e menor teor de EE $(19,21 \%)$ foi encontrado no nível estimado de $21,33 \%$ inclusão de BM. Na carne do peito observou-se deposição de 29,56\% de MS com 2,35\% de inclusão de BM e 88,56\% de deposição de PB para 13,46\% de inclusão de BM. Contudo as características físicas da carne não sofreram influência pelos níveis de inclusão do BM. Recomenda-se, com base na composição química da carne, utilizar até o nível de $20 \%$ de inclusão do BM.

Palavras-chave: Alimento alternativo, carne de frango, composição química, cor, textura

Parte da Dissertação de Mestrado do primeiro autor.

2 Prof ${ }^{a}$ M.e do Instituto Federal de Educação, Ciência e Tecnologia do Tocantins, IFTO, Dianópolis, TO. E-mail: alineamorim19@ gmail.com

3 Prof. Dr., Universidade Federal do Maranhão, UFM, Centro de Ciências Agrárias e Ambientais, Chapadinha, MA, Bolsista FAPEMA. E-mail: jc.siqueira@ufma.br

4 Profs. Drs., Escola de Medicina Veterinária e Zootecnia, Universidade Federal do Tocantins, UFT, Araguaína, TO. E-mail: rodrigueskf@mail.uft.edu.br; betagmvvaz@yahoo.com.br; silviaminharro@gmail.com; hdsantos@webmail.uft.edu.br; biana_ rosa@yahoo.com.br

5 Prof ${ }^{\mathrm{a}} \mathrm{Dr}^{\mathrm{a}}$, Instituto Federal de Educação, Ciência e Tecnologia do Tocantins, IFTO, Dianópolis, TO. E-mail: jp-zoot@hotmail.com

6 Discente do Curso de Doutorado do Programa de Pós-Graduação em Ciência Animal Tropical, UFT, Araguaína, TO. E-mail: elisson.gomes@hotmail.com

7 Prof. M.e, Universidade Paulista, UNIP, Marabá, PA. E-mail: isammom@outlook.com

8 Dr. em Ciência Animal Tropical, Araguaína, TO. E-mail: iberepereira@hotmail.com

9 Prof. Especialista em Educação Matemática, Instituto Federal de Educação, Ciência e Tecnologia do Tocantins, IFTO, Palmas, TO.E-mail: ailton.rs@hotmail.com

* Autor para correspondência 


\begin{abstract}
The purpose of this study the effect of inclusion levels $(0,10,20$ and 30\%) of cassava bagasse (BM) to the diet on the physical and chemical parameters of raw and cooked meat thigh, drumstick and breast of chicken was to evaluate cutting hillbilly kind of lineage Label rouge ${ }^{\circledR}$ slaughtered at 84 days of age . Analyzes were performed in the laboratory of Animal Nutrition, Universidade Federal do Tocantins - UFT, Escola de Medicina Veterinária e Zootecnia , Campus Araguaína . Forty chicken carcasses, which were divided into half-carcases, half-carcases and 10 for analysis of the chemical composition of raw meat and the other 10 half-carcasses for analysis of chemical composition of cooked meat, they are included in these analyzes the crude protein (CP), ether extract ( EE), dry matter (DM) and ash (CZ), with base on the percentage of dry matter, in addition to gross energy (GE) and cooking losses. Analyzes were also conducted of the physical attributes of meat (color, $\mathrm{pH}$ and texture) other 20 halfcarcasses. The inclusion of BM in the diet of broilers caipira type cut and cooking affected $(\mathrm{P}<0.05)$ the chemical characteristics of meat. Observed in thigh meat linear reduction in the deposition of $\mathrm{CZ}$ and linear increase in deposition of PB. Drumstick meat in CP content increased up to the level of $17.22 \%$ inclusion of BM corresponding to the deposition of $84.21 \% \mathrm{CP}$; and lower levels of EE (19.21\%) was found in the estimated level of $21.33 \%$ inclusion of BM. In breast meat was observed deposition of $29.56 \%$ with $2.35 \% \mathrm{DM}$ inclusion of BM deposition and $88.56 \%$ to $13.46 \%$ of CP inclusion of BM. However the physical characteristics of meat were not influenced by the levels of inclusion of BM. It is recommended, with base on the chemical composition of the meat, to use up the $20 \%$ level of inclusion of BM.
\end{abstract}

Key words: Alternative food, chicken, chemical composition, color, texture

\section{Introdução}

A avicultura é uma atividade que tem se expandido cada vez mais no Brasil, terceiro maior produtor e o primeiro em exportação, com consumo per capita de 47,38 kg de carne de frango (UBABEF, 2012). A globalização da economia e a adequação às novas tecnologias, como inovação nas instalações e equipamentos, aliados à biosseguridade nas granjas e mão de obra qualificada, têm proporcionado o sucesso à atividade.

Em meio a esta expansão da avicultura industrial, tem-se notado uma demanda crescente por produtos ditos naturais, obtidos a partir de criações ou de culturas as quais se adotam técnicas de manejo que minimizem a utilização de produtos artificiais, pois os produtos naturais estão aliados à imagem de produtos saudáveis e que atendem os padrões de bem-estar animal. Esta é uma realidade que abre as portas para a avicultura alternativa, administrada geralmente por pequenos produtores que sobrevivem da agricultura familiar.

Segundo Brasil (1999) as aves criadas em sistema alternativo ou aves caipiras devem se alimentar com dietas exclusivamente vegetais, devendo após os 25 dias de idade ter acesso a piquete, sendo a idade mínima de abate de 85 dias.

Dentre as linhagens de frango caipira, destacase a linhagem Label rouge ${ }^{\circledR}$, que possui maior aceitabilidade por grande parte dos produtores com maior adaptabilidade ao clima tropical (ZANUSSO; DIONELLO, 2003). É uma ave de crescimento lento, conhecida popularmente como pescoço pelado, atinge peso de abate $(2,5 \mathrm{~kg})$ por volta dos 90 dias de idade.

Sabe-se que a alimentação entra como grande parte do custo de produção na criação das aves, chegando a aproximadamente $75 \%$ do custo total. Entre as matérias-primas comumente utilizadas estão o milho e o farelo de soja que representam a maior parcela na composição das rações. No entanto, durante a entressafra essas matérias primas tornam-se onerosas. Com isso, atualmente busca-se alternativas para esses ingredientes com o principal objetivo de baratear o custo da ração sem deixar de fornecer os nutrientes essenciais para o bom desempenho das aves. 
A mandioca (Manihot esculenta Crantz) e seus resíduos podem ser fontes alternativas de energia. Apesar de parte de sua produção ser utilizada na alimentação humana, seus resíduos como a raspa ou o bagaço resultante da extração da fécula da mandioca, podem ser utilizados com eficiência na alimentação animal (MARQUES et al., 2000; SOUSA et al., 2012).

Os subprodutos apresentam valores, tanto qualitativos, como quantitativos bastante variáveis, devido a fatores como: cultivar e idade da planta, tempo após a colheita, regulagem das máquinas de colheita e forma de processamento utilizada pela indústria.

As características que tornam a carne de frango caipira um produto atrativo ao consumidor, além da própria forma de criação, são os parâmetros físicos e químicos. Os parâmetros físicos comumente avaliados são a cor, a textura e o tempo de cozimento; enquanto que nutricionalmente são avaliados os teores de lipídio e proteína na carne; onde o frango caipira criado em sistema alternativo possui características diferenciadas daqueles da avicultura industrial.

Diante do exposto, objetivou-se avaliar o efeito da inclusão do bagaço de mandioca nas rações sobre os parâmetros físicos e químicos da carne crua e cozida da coxa, sobrecoxa e peito de frangos tipo caipira da linhagem Label rouge ${ }^{\circledR}$.

\section{Material e Métodos}

O experimento foi conduzido no setor de avicultura e as análises de composição química da carne crua e cozida foram realizadas no laboratório de nutrição da Universidade Federal do Tocantins UFT, Centro de Ciência Animal Tropical, campus de Araguaína - Tocantins.
Foram utilizadas 280 aves de crescimento lento, machos, da linhagem Label rouge ${ }^{\circledR}$ (pescoço pelado). Até o sétimo dia de idade os pintos receberam ração a base de milho e farelo de soja, formulada para atender as exigências nutricionais de cada fase de acordo com as recomendações de Rostagno et al. (2011).

$\mathrm{Na}$ fase inicial (8 a 28 dias) as aves foram distribuídas, em delineamento inteiramente casualizado, com quatro tratamentos, cinco repetições e quatorze aves por repetição, em baterias providas de 20 gaiolas metálicas $(1,00 \times 1,00 \times 0,40 \mathrm{~m})$, equipadas com comedouros e bebedouros tipo calha, sistema de iluminação e aquecimento. O galpão dispunha de cortinas laterais manejadas de acordo com a temperatura e o comportamento das aves.

Nas fases de crescimento (29 a 56 dias) e final (56 a 84 dias) as aves foram transferidas, respeitando a distribuição dos tratamentos da fase inicial, em uma área constituída de 20 piquetes, cada um dispondo de um abrigo e uma área de pastejo. Cada abrigo possuía pé-direito de $1,5 \mathrm{~m}$ coberto com palha de babaçu, sem laterais, construído de madeira, e área útil de $2 \mathrm{~m}^{2}$, onde localizavam-se um comedouro tubular e um bebedouro do tipo pendular. A área de pastejo, cercada por tela galvanizada, possuía uma área total de $50 \mathrm{~m}^{2}$, contendo predominantemente gramíneas Cynodon sp.

Em cada fase de criação os tratamentos consistiram em rações formuladas para atender as exigências nutricionais das aves segundo Rostagno et al. (2011) com os níveis de 0,10,20,30\% de inclusão do bagaço da mandioca. A composição bromatológica dos principais ingredientes utilizados na formulação das rações experimentais estão apresentadas na Tabela 1, e a composição percentual e química das rações experimentais para as fases: inicial, crescimento e final estão expressas nas Tabelas 2, 3 e 4, respectivamente. 
Tabela 1. Composição dos ingredientes utilizados na formulação das rações experimentais (com base na matéria natural).

\begin{tabular}{lccc}
\hline \multicolumn{1}{c}{ Nutriente } & Milho $^{1}$ & Farelo de Soja $^{1}$ & Bagaço da Mandioca $^{\circ}$ \\
\hline Energia Metabolizável (kcal/kg) & 3381 & 2256 & $2508^{2}$ \\
Proteína bruta (\%) & 8,26 & 45,32 & $1,26^{2}$ \\
Extrato Etéreo (\%) & 3,65 & 1,69 & $3,86^{2}$ \\
Cálcio (\%) & 0,030 & 0,240 & $0,200^{1}$ \\
Fibra Bruta (\%) & 1,73 & 5,30 & $11,1^{2}$ \\
Fósforo disponível (\%) & 0,080 & 0,180 & $0,300^{1}$ \\
Cloro (\%) & 0,050 & 0,050 & $0,050^{1}$ \\
Potássio (\%) & 0,280 & 1,830 & $\mathrm{ND}^{3}$ \\
Sódio (\%) & 0,020 & 0,020 & $0,030^{1}$ \\
Lisina total (\%) & 0,240 & 2,770 & $0,090^{1}$ \\
Metionina total (\%) & 0,170 & 0,640 & $0,030^{1}$ \\
Met+cistina total (\%) & 0,360 & 1,370 & $0,070^{1}$ \\
Treonina total (\%) & 0,320 & 1,780 & $0,070^{1}$ \\
Triptofano total (\%) & 0,070 & 0,620 & $0,020^{1}$ \\
\hline
\end{tabular}

${ }^{1}$ Rostagno et al. (2005); ${ }^{2}$ Laboratório Universidade Federal do Tocantins ${ }^{3}$ Não determinado.

Fonte: Elaboração dos autores.

Tabela 2. Composição percentual das rações experimentais para a fase inicial ( 8 a 28 dias).

\begin{tabular}{lllll}
\hline \multirow{2}{*}{ Ingredientes } & \multicolumn{4}{c}{ Níveis de inclusão do bagaço de mandioca (\%) } \\
\cline { 2 - 5 } Milho & 59,850 & 46,011 & 32,171 & 30 \\
Farelo de Soja (45\%) & 33,763 & 35,858 & 37,952 & 19,288 \\
Bagaço de mandioca & 0,0000 & 10,0000 & 20,000 & 39,198 \\
Óleo de soja & 2,451 & 4,336 & 6,221 & 7,956 \\
Fosfato bicálcico & 1,802 & 1,660 & 1,518 & 1,383 \\
Calcário & 0,921 & 0,958 & 0,995 & 1,032 \\
Sal comum & 0,494 & 0,492 & 0,490 & 0,489 \\
DL-Metionina & 0,200 & 0,212 & 0,224 & 0,244 \\
L-Lisina HCl & 0,109 & 0,063 & 0,018 & 0,0000 \\
Núcleo1 & 0,4000 & 0,4000 & 0,4000 & 0,4000 \\
BHT & 0,0100 & 0,0100 & 0,0100 & 0,0100 \\
Total & 100,000 & 100,000 & 100,000 & 100,000 \\
\hline & Composição nutricional calculada & 3000 \\
\hline EM (kcal/kg) & 3000 & 3000 & 3000 & 19,624 \\
Proteína bruta (\%) & 20,077 & 20,029 & 19,965 & 10,444 \\
Extrato Etéreo (\%) & 5,190 & 6,982 & 8,773 & 0,8878 \\
Cálcio (\%) & 0,8878 & 0,8878 & 0,8878 & 5,7379 \\
Fibra bruta (\%) & 2,8248 & 3,8057 & 4,7865 & 0,4436 \\
Fósforo disponível (\%) & 0,4436 & 0,4436 & 0,4436 & 0,3373 \\
Cloro (\%) & 0,3470 & 0,3437 & 0,3405 & 0,7729 \\
Potássio (\%) & 0,7914 & 0,7897 & 0,7879 & 0,2147 \\
Sódio (\%) & 0,2147 & 0,2147 & 0,2147 & 1,1650 \\
Lisina total (\%) & 1,1650 & 1,1650 & 1,1650 & 0,5298 \\
Metionina total (\%) & 0,5381 & 0,5336 & 0,5292 & 0,8275 \\
Metionina + cistina total (\%) & 0,8275 & 0,8275 & 0,8275 & 0,7800 \\
Treonina total (\%) & 0,7925 & 0,7925 & 0,7925 & 0,2585 \\
Triptofano total (\%) & 0,2486 & 0,2536 & 0,2585 & \\
\hline
\end{tabular}

${ }^{1}$ Composição/tonelada: Manganês 18,1750 mg, zinco 17,500 mg, ferro 11,250, cobre $2000 \mathrm{mg}$, iodo 187,50 mg, selênio $75 \mathrm{mg}$, vitamina K3 $360 \mathrm{mg}$, vitamina B1 436,50 mg, vitamina B2 $4300 \mathrm{mg}$, vitamina B6 $624 \mathrm{mg}$, vitamina B12 $2400 \mathrm{mg}$, ácido fólico $200 \mathrm{mg}$, ácido pantotênico 3,120 mg, niacina 8,400 mg, biotina 10,000 mg, colina 78,102,01 mg, aditivo antioxidante 25,000 mg.

Fonte: Elaboração dos autores. 
Tabela 3. Composição percentual das rações experimentais para a fase de crescimento ( 29 a 56 dias).

\begin{tabular}{lcccc}
\hline \multirow{2}{*}{ Ingredientes } & \multicolumn{4}{c}{ Níveis de inclusão do bagaço de mandioca (\%) } \\
\cline { 2 - 5 } & 0 & 10 & 20 & 30 \\
\hline Milho & 71,282 & 57,253 & 43,225 & 30,139 \\
Farelo de Soja (45\%) & 24,468 & 26,730 & 28,993 & 30,419 \\
Bagaço de mandioca & 0,000 & 10,000 & 20,000 & 30,000 \\
Óleo de soja & 1,006 & 2,921 & 4,836 & 6,603 \\
Fosfato bicálcico & 1,394 & 1,250 & 1,107 & 0,970 \\
Calcário & 0,768 & 0,805 & 0,842 & 0,879 \\
Sal comum & 0,413 & 0,412 & 0,410 & 0,408 \\
DL-Metionina & 0,133 & 0,143 & 0,154 & 0,172 \\
L-Lisina HCl & 0,126 & 0,075 & 0,024 & 0,0000 \\
Núcleo1 & 0,4000 & 0,4000 & 0,4000 & 0,4000 \\
BHT & 0,0100 & 0,0100 & 0,0100 & 0,0100 \\
Total & 100,000 & 100,000 & 100,000 & 100,000 \\
\hline & Composição nutricional calculada & & \\
\hline EM (kcal/kg) & 3050 & 3050 & 3050 & 3050 \\
Proteína bruta (\%) & 16,7890 & 16,7890 & 16,7890 & 16,7890 \\
Extrato Etéreo (\%) & 4,011 & 5,816 & 7,642 & 9,344 \\
Cálcio (\%) & 0,7113 & 0,7113 & 0,7113 & 0,7113 \\
Fibra bruta (\%) & 2,5300 & 3,5164 & 4,5029 & 5,4603 \\
Fósforo disponível (\%) & 0,3545 & 0,3545 & 0,3545 & 0,3545 \\
Cloro (\%) & 0,3013 & 0,2980 & 0,2947 & 0,2916 \\
Potássio (\%) & 0,6545 & 0,6552 & 0,6559 & 0,6437 \\
Sódio (\%) & 0,1832 & 0,1832 & 0,1832 & 0,1832 \\
Lisina total (\%) & 0,9450 & 0,9450 & 0,9450 & 0,9450 \\
Metionina total (\%) & 0,4423 & 0,4370 & 0,4316 & 0,4310 \\
Metionina + cistina total (\%) & 0,6800 & 0,6800 & 0,6800 & 0,6800 \\
Treonina total (\%) & 0,6636 & 0,6660 & 0,6684 & 0,6585 \\
Triptofano total (\%) & 0,1969 & 0,2028 & 0,2087 & 0,2097 \\
\hline Compoco & & & \\
\hline
\end{tabular}

${ }^{1}$ Composição/tonelada: Manganês $18,1750 \mathrm{mg}$, zinco 17,500 mg, ferro 11,250, cobre $2000 \mathrm{mg}$, iodo $187,50 \mathrm{mg}$, selênio $75 \mathrm{mg}$, vitamina K3 360 mg, vitamina B1 436,50 mg, vitamina B2 4300 mg, vitamina B6 624 mg, vitamina B12 2400 mg, ácido fólico $200 \mathrm{mg}$, ácido pantotênico 3,120 mg, niacina 8,400 mg, biotina 10,000 mg, colina 78,102,01 mg, aditivo antioxidante $25,000 \mathrm{mg}$.

Fonte: Elaboração dos autores.

Aos 84 dias de idade as aves foram submetidas a jejum alimentar por 12 horas, sendo amostradas duas aves de cada parcela, totalizando 40 aves, com peso corporal próximo à média da parcela $( \pm 10 \%)$. As aves foram individualmente identificadas e abatidas por deslocamento cervical, depenadas e evisceradas, em seguida acondicionadas em sacos plásticos e congeladas a $-18^{\circ} \mathrm{C}$ para posteriores análises.

As dez carcaças selecionadas de cada tratamento, totalizando 40 carcaças, foram descongeladas em geladeira a $4^{\circ} \mathrm{C}$ por 24 horas, divididas ao meio, totalizando 80 meias carcaças. Vinte meias carcaças foram utilizadas para análise da composição química da carne crua e outras 20 meias carcaças da mesma ave foram utilizadas para análise da composição química da carne cozida. Logo após a divisão das carcaças em meias carcaças, foram separados os cortes de coxa, sobrecoxa e peito, os quais foram desossados e retirados a pele, gordura e ligamentos para realização das análises de composição química da carne crua e cozida, sendo o restante dos componentes da carcaça descartados.

Os cortes da carne crua da coxa, sobrecoxa e 
peito foram triturados separadamente em cutter, pré-secos em estufa a $55^{\circ} \mathrm{C}$ e pré-desengordurados em aparelho soxhlet para retirar o excesso de água e gordura. Em seguida as amostras foram trituradas em moinho tipo faca em peneira $1 \mathrm{~mm}$ e submetidas a análises para determinação de sua composição em proteína bruta, extrato etéreo, cinzas, matéria seca, umidade e energia bruta. As metodologias utilizadas para as análises são descritas por Silva e Queiroz (2006). Os teores de água e gordura retirados na pré-secagem e pré-desengorduramento foram considerados para correção dos valores nas análises subsequentes.

Tabela 4. Composição percentual das rações experimentais para a fase final (57 a 84 dias).

\begin{tabular}{|c|c|c|c|c|}
\hline \multirow{2}{*}{ Ingredientes } & \multicolumn{4}{|c|}{ Níveis de inclusão do bagaço de mandioca (\%) } \\
\hline & 0 & 10 & 20 & 30 \\
\hline Milho & 77,407 & 63,379 & 49,350 & 35,511 \\
\hline Farelo de Soja (45\%) & 19,593 & 21,856 & 24,118 & 26,212 \\
\hline Bagaço de mandioca & 0,000 & 10,000 & 20,000 & 30,000 \\
\hline Óleo de soja & 0,469 & 2,384 & 4,229 & 6,184 \\
\hline Fosfato bicálcico & 0,609 & 0,790 & 0,647 & 0,504 \\
\hline Calcário & 0,609 & 0,646 & 0,683 & 0,720 \\
\hline Sal comum & 0,331 & 0,329 & 0,327 & 0,326 \\
\hline DL-Metionina & 0,100 & 0,110 & 0,121 & 0,133 \\
\hline L-Lisina $\mathrm{HCl}$ & 0,147 & 0,096 & 0,045 & 0,000 \\
\hline Núcleo1 & 0,400 & 0,400 & 0,400 & 0,400 \\
\hline BHT & 0,010 & 0,010 & 0,010 & 0,010 \\
\hline Total & 100,000 & 100,000 & 100,000 & 100,000 \\
\hline \multicolumn{5}{|c|}{ Composição nutricional calculada } \\
\hline EM (kcal/kg) & 3100 & 3100 & 3100 & 3100 \\
\hline Proteína bruta (\%) & 15,0860 & 15,0860 & 15,0860 & 15,0860 \\
\hline Extrato Etéreo (\%) & 3,61 & 5,426 & 7,182 & 9,038 \\
\hline Cálcio (\%) & 0,5286 & 0,5286 & 0,5286 & 0,5286 \\
\hline Fibra bruta $(\%)$ & 2,3776 & 3,3641 & 4,3505 & 5,3303 \\
\hline Fósforo disponível (\%) & 0,2623 & 0,2623 & 0,2623 & 0,2623 \\
\hline Cloro (\%) & 0,2534 & 0,2501 & 0,2468 & 0,2435 \\
\hline Potássio (\%) & 0,5830 & 0,5838 & 0,5846 & 0,5823 \\
\hline Sódio (\%) & 0,1507 & 0,1507 & 0,1507 & 0,1507 \\
\hline Lisina total $(\%)$ & 0,8400 & 0,8400 & 0,8400 & 0,8400 \\
\hline Metionina total (\%) & 0,3944 & 0,3890 & 0,3836 & 0,3794 \\
\hline Metionina + cistina total $(\%)$ & 0,6050 & 0,6050 & 0,6050 & 0,6050 \\
\hline Treonina total $(\%)$ & 0,5965 & 0,5988 & 0,6012 & 0,6008 \\
\hline Triptofano total $(\%)$ & 0,1699 & 0,1757 & 0,1817 & 0,1864 \\
\hline
\end{tabular}

${ }^{1}$ Composição/tonelada: Manganês 18,1750 mg, zinco 17,500 mg, ferro 11,250, cobre $2000 \mathrm{mg}$, iodo $187,50 \mathrm{mg}$, selênio $75 \mathrm{mg}$, vitamina K3 360 mg, vitamina B1 436,50 mg, vitamina B2 $4300 \mathrm{mg}$, vitamina B6 $624 \mathrm{mg}$, vitamina B12 $2400 \mathrm{mg}$, ácido fólico $200 \mathrm{mg}$, ácido pantotênico 3,120 mg, niacina $8,400 \mathrm{mg}$, biotina 10,000 mg, colina 78,102,01 mg, aditivo antioxidante $25,000 \mathrm{mg}$. Fonte: Elaboração dos autores.

$\mathrm{Na}$ análise da carne cozida as amostras passaram pelo mesmo procedimento, com a diferença do processo de cocção, no qual as amostras foram envolvidas separadamente em papel alumínio e colocadas para cozer em chapa pré-aquecida a $150^{\circ} \mathrm{C}$. Ao atingir temperatura interna de $35^{\circ} \mathrm{C}$ foram viradas e mantidas até atingir temperatura interna de $72^{\circ} \mathrm{C}$ e em seguida resfriadas a temperatura ambiente. Após esse procedimento as análises foram conduzidas da mesma forma que na carne crua, para 
realização da composição química na carne cozida. Foi possível também calcular a perda por cozimento (PPC) pela média das diferenças de peso entre as amostras antes e depois do cozimento, resfriadas em temperatura ambiente de acordo com Ramos e Gomide (2007).

Em outras 20 meias carcaças foram avaliadas nos cortes da carne crua da coxa, sobrecoxa e peito, sem osso, pele, ligamentos e gordura, a coloração da carne pelo sistema CIELAB ( $\mathrm{L}^{*}=$ Luminosidade, $\mathrm{a}^{*}=$ teor de vermelho e $\mathrm{b}^{*}=$ teor de amarelo) com colorímetro (Chroma meter), sendo a leitura realizada em três pontos distintos da musculatura, utilizando-se os valores médios. Também foi coletado, sequencialmente, nesses mesmos cortes os valores de $\mathrm{pH}$.

Nas 20 meias carcaças restantes foi avaliada a força de cisalhamento, após passarem pelo processo de cocção. De cada amostra foram extraídas quatro alíquotas, no sentido da fibra, com auxílio de um molde cilíndrico $(1,27 \mathrm{~cm}$ de diâmetro). A força de cisalhamento (kgf) foi medida pela secção dos pedaços, usando o aparelho texturômetro acoplado a lâmina Warner-Bratzler Shear Force, sendo utilizados os valores médios das quatro alíquotas de cada amostra.

Os dados de cada variável avaliada na carne crua e cozida foram submetidos à testes de normalidade (Cramer-Von Mises) e homocedasticidade (Levene). Para as variáveis medidas na carne crua e cozida, para explorar o efeito do cozimento, a análise foi realizada sob o esquema fatorial $(4 \times 2)$ de acordo com o modelo estatístico:

$$
\mathrm{y}_{\mathrm{ijk}}=\mu+\mathrm{Coz}_{\mathrm{i}}+\mathrm{NBM}_{\mathrm{j}}+\mathrm{Coz}^{*} \mathrm{NBM}_{\mathrm{ij}}+\mathrm{e}_{\mathrm{ijk}} ;
$$

Em que, $y_{\mathrm{ijk}}=$ valor da variável dependente correspondente ao cozimento i com o nível de bagaço de mandioca $\mathrm{j}$ na k-ésima repetição; $\mathrm{Coz}_{\mathrm{i}}=$ efeito do cozimento; $\mathrm{NBM}_{\mathrm{j}}=$ efeito do i-ésimo nível do bagaço de mandioca na ração; $\mathrm{Coz}^{*} \mathrm{NBM}_{\mathrm{ij}}=$ efeito da interação entre o cozimento e o nível de bagaço de mandioca na ração; $\mathrm{e}_{\mathrm{ijk}}=$ erro experimental.

Quando os efeitos do NBM sobre os componentes químicos da carne foram detectados pela análise de variância, os efeitos foram explorados por análise de regressão por meio de modelos polinomiais de primeira ou segunda ordem. No caso de interação significativa pelo teste $\mathrm{F}(\mathrm{P}<0,05)$, os modelos foram ajustados aos dados da carne crua e da carne cozida isoladamente, no caso de interação não significativa $(\mathrm{P}>0,05)$ os modelos foram ajustados ao conjunto de dados da carne crua e cozida.

Já os dados das características físicas avaliadas na carne crua e as perdas por cozimento foram submetidos à testes de normalidade (Cramer-Von Mises) e homocedasticidade (Levene). Atendidas estas pressuposições foram submetidos à análise de variância segundo o modelo estatístico: $\mathrm{Y}_{\mathrm{ij}}$ $=\mu+\mathrm{NBM}_{\mathrm{i}}+\mathrm{e}_{\mathrm{ij}}$. Em que: $\mathrm{Y}_{\mathrm{ij}}=$ valor da j-ésima observação para variável dependente no i-ésimo nível de inclusão do bagaço da mandioca; $\mu=$ média geral comum a todas as observações; $\mathrm{NBM}_{\mathrm{i}}$ = efeito do i-ésimo nível de inclusão do bagaço da mandioca, e $\mathrm{e}_{\mathrm{ij}}=$ erro experimental.

Posteriormente, os dados foram submetidos a análises de regressão por meio de modelos polinomiais de primeira e segunda ordem, considerando-se para o ajuste dos modelos o nível de significância do teste $\mathrm{F}$ e o coeficiente de determinação $\left(\mathrm{R}^{2}\right)$. Para as análises estatísticas utilizou-se o software SAS 9.0 (STATISTICAL ANALYSIS SYSTEM, 2002).

\section{Resultados e Discussão}

Análises químicas da carne da coxa

Houve interação significativa $(\mathrm{P}<0,05)$ entre cocção (COC) e níveis de inclusão de bagaço de mandioca (NBM) para o teor de cinzas. O teor de cinzas (CZ) foi influenciado pelos níveis de inclusão de BM apresentando redução linear na carne crua: $\mathrm{CZ}=4,26-0,015 \mathrm{NBM}\left(\mathrm{P}=0,019 ; \mathrm{r}^{2}=0,45\right)$, porém não houve efeito dos NBM na carne cozida. Nas demais variáveis, matéria seca (MS), proteína bruta (PB), extrato etéreo (EE) e energia bruta (EB), não houve interação significativa entre COC x NBM $(\mathrm{P}>0,05)$ (Tabela 5). 
Tabela 5. Análises químicas da carne da coxa crua e cozida de frangos caipiras alimentado com níveis crescentes de inclusão de bagaço de mandioca.

\begin{tabular}{|c|c|c|c|c|c|c|c|c|c|c|}
\hline \multirow[t]{2}{*}{ Variável } & \multirow{2}{*}{$\begin{array}{l}\text { Cocção } \\
\text { (COC) }\end{array}$} & \multicolumn{4}{|c|}{$\begin{array}{l}\text { Nível de bagaço de mandioca } \\
(\mathrm{NBM})(\%)\end{array}$} & \multirow[t]{2}{*}{ Geral } & \multirow[t]{2}{*}{$\mathrm{CV}^{1}$} & \multicolumn{3}{|c|}{$\mathrm{P}>\mathrm{F}^{2}$} \\
\hline & & 0 & 10 & 20 & 30 & & & $\mathrm{COC}$ & NBM & COC $\times$ NBM \\
\hline \multirow{3}{*}{ MS (\%) } & Sim & 31,89 & 29,12 & 30,69 & 29,64 & 30,33 & \multirow{3}{*}{5,66} & \multirow{3}{*}{$<0,001$} & \multirow{3}{*}{0,034} & \multirow{3}{*}{0,736} \\
\hline & Não & 26,23 & 24,99 & 25,50 & 24,92 & 25,40 & & & & \\
\hline & $\mathrm{Geral}^{3}$ & $29,06^{\mathrm{a}}$ & $27,05^{\mathrm{b}}$ & $28,38^{\mathrm{ab}}$ & $27,28^{\mathrm{b}}$ & & & & & \\
\hline \multirow{3}{*}{ PB (\%) } & Sim & 71,00 & 70,41 & 74,93 & 78,03 & 73,49 & \multirow{3}{*}{5,01} & \multirow{3}{*}{$<0,001$} & \multirow{3}{*}{0,027} & \multirow{3}{*}{0,096} \\
\hline & Não & 76,58 & 82,32 & 80,35 & 81,11 & 80,07 & & & & \\
\hline & Geral $^{4}$ & 74,10 & 76,36 & 77,34 & 79,74 & & & & & \\
\hline \multirow{3}{*}{ EE (\%) } & Sim & 12,61 & 16,35 & 17,99 & 16,64 & 15,89 & \multirow{3}{*}{17,54} & \multirow{3}{*}{0,911} & \multirow{3}{*}{0,065} & \multirow{3}{*}{0,310} \\
\hline & Não & 15,16 & 14,87 & 16,23 & 16,92 & 15,77 & & & & \\
\hline & Geral & 13,89 & 15,61 & 17,21 & 16,78 & & & & & \\
\hline \multirow{3}{*}{ CZ (\%) } & $\operatorname{Sim}^{\mathrm{NS}}$ & 3,37 & 3,58 & 3,34 & 3,49 & 3,45 & \multirow{3}{*}{7,07} & \multirow{3}{*}{$<0,001$} & \multirow{3}{*}{0,177} & \multirow{3}{*}{0,005} \\
\hline & Não & 4,30 & 3,92 & 4,29 & 3,70 & 4,04 & & & & \\
\hline & Geral & 3,84 & 3,75 & 3,76 & 3,59 & & & & & \\
\hline \multirow{3}{*}{$\begin{array}{c}\text { EB (kcal/ } \\
\mathrm{kg})\end{array}$} & Sim & 5132 & 4907 & 4605 & 4796 & 4860 & \multirow{3}{*}{6,28} & \multirow{3}{*}{$<0,001$} & \multirow{3}{*}{0,526} & \multirow{3}{*}{0,132} \\
\hline & Não & 5464 & 5642 & 5672 & 5448 & 4811 & & & & \\
\hline & Geral & 5298 & 5274 & 5079 & 5122 & & & & & \\
\hline
\end{tabular}

${ }^{1}$ Coeficiente de variação (\%); ${ }^{2}$ Significância do teste $\mathrm{F}$ da análise de variância; ${ }^{3}$ Nenhum do modelos de regressão se ajustou aos dados $(\mathrm{P}>0,05)$, médias seguida de letra iguais minúsculas não diferem pelo teste $\mathrm{SNK}(\mathrm{P}>0,05)$; ${ }^{4} \mathrm{Efeito}$ linear: $\mathrm{PB}=$ $74,21+0,179 \mathrm{NBM}\left(\mathrm{P}=0,027 ; \mathrm{r}^{2}=0,97\right), \mathrm{CZ}=4,26-0,015 \mathrm{NBM}\left(\mathrm{P}=0,019 ; \mathrm{r}^{2}=0,45\right)$. ${ }^{\text {NS }}$ Não significativo $(\mathrm{P}>0,05)$.

Fonte: Elaboração dos autores.

A redução linear no teor de $\mathrm{CZ}$ na carne crua com aumento dos níveis de inclusão BM pode ser explicado de acordo com Raupp et al. (2002), que avaliaram a influência da fibra do bagaço de mandioca em um estudo realizado com ratos recebendo dietas com, acréscimo de 5,15 e $25 \%$ de bagaço de mandioca substituindo igual quantidade de amido na dieta base. Os resultados apontaram que o bagaço de mandioca hidrolisado promoveu arraste significativo de minerais e proteína e que a partir do acréscimo de 15 e $25 \%$ de BM houve também o arraste de lipídeos e carboidratos digestíveis.

Os níveis de inclusão de bagaço de mandioca (BM) influenciaram no teor de $\mathrm{MS}(\mathrm{P}<0,05)$, onde a ração sem inclusão de $\mathrm{BM}$ apresentou maior teor de MS diferindo estatisticamente do nível de $10 \%$ e $30 \%$ de inclusão de BM que apresentaram menores teores de MS. Com maior arraste fecal de minerais consequentemente menos minerais serão depositados no músculo. Esse arraste de nutrientes causados pela ingestão de fibras do BM também pode ter sido o motivo do menor teor de MS no músculo.

Os níveis de inclusão de BM também influenciaram o teor de $\mathrm{PB}$ apresentando aumento linear: $\mathrm{PB}=74,21+0,179 \mathrm{NBM}\left(\mathrm{P}=0,027 ; \mathrm{r}^{2}=0,97\right)$. Como o BM é um alimento rico em fibra e amido, estes podem ter contribuído para uma maior retenção de $\mathrm{PB}$ na musculatura, pois de acordo com o trabalho de Feniman (2004) o teor de amido e fibras solúveis aumentou significativamente com o aumento do período de colheita da mandioca de 12 meses para 15 meses de idade da planta, como não se sabe o período de colheita da mandioca do presente trabalho, acredita-se que a mandioca teve um maior período de colheita implicando num BM com alta concentração de amido e com fibra de maior solubilidade.

Comportamento semelhantes foi encontrado no trabalho de Arruda et al. (2003), que avaliaram diferentes níveis de amido (22 e $32 \%$ ) e fontes de 
fibra (feno de alfafa e casca de soja) na ração de coelhos e verificaram uma tendência de retenção energética e proteica na carcaça desses animais em dietas contendo maior nível de amido e fibra de alta solubilidade.

\section{Análises químicas da carne da sobrecoxa}

A interação entre COC x NBM foi significativa $(\mathrm{P}<0,05)$ para o teor de $\mathrm{PB}, \mathrm{EE}$ e EB. Observou- se efeito quadrático no teor de PB na carne cozida: $\mathrm{PB}=77,91+0,93 \mathrm{NBM}-0,027 \mathrm{NBM}^{2} \quad(\mathrm{P}<0,001$; $\left.\mathrm{R}^{2}=0,82\right)$, onde o nível estimado de $17,22 \%$ de inclusão de bagaço de mandioca refletiu em $84,21 \%$ de deposição de proteína na sobrecoxa. $\mathrm{Na}$ carne crua houve efeito linear crescente: $\mathrm{PB}=78,48-$ $0,102 \mathrm{NBM}\left(\mathrm{P}=0,004 \mathrm{r}^{2}=0,97\right)$ (Tabela 6).

Tabela 6. Análises químicas da carne da sobrecoxa crua e cozida de frangos caipiras alimentados com níveis crescentes de bagaço de mandioca.

\begin{tabular}{|c|c|c|c|c|c|c|c|c|c|c|}
\hline \multirow[t]{2}{*}{ Variável } & \multirow{2}{*}{$\begin{array}{l}\text { Cocção } \\
\text { (COC) }\end{array}$} & \multicolumn{4}{|c|}{$\begin{array}{l}\text { Nível de bagaço de mandioca } \\
(\mathrm{NBM})(\%)\end{array}$} & \multirow[t]{2}{*}{ Geral } & \multirow[t]{2}{*}{$\mathrm{CV}^{1}$} & \multicolumn{3}{|c|}{$\mathrm{P}>\mathrm{F}^{2}$} \\
\hline & & 0 & 10 & 20 & 30 & & & $\mathrm{COC}$ & NBM & $\mathrm{COC} \times \mathrm{NBM}$ \\
\hline \multirow{3}{*}{ MS (\%) } & Sim & 32,58 & 32,66 & 31,53 & 32,29 & 32,30 & \multirow{3}{*}{5,24} & \multirow{3}{*}{$<0,001$} & \multirow{3}{*}{0,592} & \multirow{3}{*}{0,570} \\
\hline & Não & 25,98 & 26,77 & 26,48 & 25,27 & 26,15 & & & & \\
\hline & Geral & 29,28 & 29,71 & 29,00 & 29,17 & & & & & \\
\hline \multirow{3}{*}{ PB (\%) } & $\mathrm{Sim}^{3}$ & 78,31 & 77,73 & 76,47 & 75,34 & 76,96 & \multirow{3}{*}{2,18} & \multirow{3}{*}{$<0,001$} & \multirow{3}{*}{$<0,001$} & \multirow{3}{*}{$<0,001$} \\
\hline & $\mathrm{Não}^{4}$ & 78,59 & 82,92 & 87,85 & 81,03 & 82,62 & & & & \\
\hline & Geral & 78,43 & 80,33 & 81,53 & 77,87 & & & & & \\
\hline \multirow{3}{*}{ EE (\%) } & $\mathrm{Sim}^{3}$ & 19,46 & 20,62 & 24,34 & 22,14 & 21,78 & \multirow{3}{*}{4,36} & \multirow{3}{*}{0,325} & \multirow{3}{*}{$<0,001$} & \multirow{3}{*}{0,036} \\
\hline & $\mathrm{Não}^{4}$ & 18,86 & 20,95 & 22,35 & 23,01 & 21,34 & & & & \\
\hline & Geral & 19,12 & 20,79 & 23,48 & 22,62 & & & & & \\
\hline \multirow{3}{*}{ CZ (\%) } & Sim & 3,23 & 3,37 & 3,25 & 3,08 & 3,23 & \multirow{3}{*}{7,52} & \multirow{3}{*}{$<0,001$} & \multirow{3}{*}{0,018} & \multirow{3}{*}{0,199} \\
\hline & Não & 4,05 & 3,92 & 3,51 & 3,58 & 3,79 & & & & \\
\hline & Geral $^{4}$ & 3,64 & 3,64 & 3,35 & 3,33 & & & & & \\
\hline \multirow{3}{*}{$\begin{array}{c}\mathrm{EB} \\
(\mathrm{kcal} / \mathrm{k})\end{array}$} & $\mathrm{Sim}^{4}$ & 4518 & 4171 & 4218 & 3294 & 4039 & \multirow{3}{*}{10,46} & \multirow{3}{*}{$<0,001$} & \multirow{3}{*}{0,209} & \multirow{3}{*}{0,006} \\
\hline & NãoNS & 4833 & 5527 & 5642 & 5561 & 5408 & & & & \\
\hline & Geral & 4676 & 4925 & 5032 & 4553 & & & & & \\
\hline
\end{tabular}

${ }^{1}$ Coeficiente de variação (\%); ${ }^{2}$ Significância do teste $\mathrm{F}$ da análise de variância; ${ }^{3}$ Efeito quadrático: $\mathrm{PB}=77,91+0,93 \mathrm{NBM}-$ $0,027 \mathrm{NBM}^{2}\left(\mathrm{P}<0,001 ; \mathrm{R}^{2}=0,82\right), \mathrm{EE}=18,90+0,384 \mathrm{NBM}-0,009 \mathrm{NBM}^{2}\left(\mathrm{P}=0,029 \mathrm{R}^{2}=0,71\right) ;{ }^{4}$ Efeito linear: $\mathrm{PB}=78,48-$ $0,102 \mathrm{NBM}\left(\mathrm{P}=0,004 \mathrm{r}^{2}=0,97\right), \mathrm{EE}=19,22+0,135 \mathrm{NBM}\left(\mathrm{P}<0,001 \mathrm{r}^{2}=0,95\right), \mathrm{CZ}=4,05-0,018 \mathrm{NBM}\left(\mathrm{P}=0,005 \mathrm{r}^{2}=0,86\right), \mathrm{EB}=4584$ $-37,20 \mathrm{NBM}\left(\mathrm{P}=0,003 \mathrm{r}^{2}=0,83\right)$. ${ }^{\mathrm{NS}}$ Não significativo $(\mathrm{P}>0,05)$.

Fonte: Elaboração dos autores.

Os resultados da $\mathrm{PB}$ na sobrecoxa foram semelhantes aos resultados encontrados na coxa, ocorrendo aumento no teor de PB com a inclusão de BM, podendo também ser explicado de acordo com Feniman (2004) e Arruda et al. (2003) incorrendo em maior retenção proteica na carne devido a maior quantidade de amido e solubilidade da fibra no BM.
O bagaço de mandioca também foi utilizado por Adeyemi et al. (2008), na avaliação da retenção de nutrientes na ração de frangos de corte onde utilizaram o BM juntamente com filtrado de rumem na ração farelada e peletizada de frangos de corte, o aumento dos níveis de bagaço de mandioca na ração diminuiu a retenção de proteína bruta, fibra bruta e extrato etéreo da ração. No entanto, houve influencia 
dos níveis de inclusão do bagaço de mandioca na ração sobre a forma peletizada que favoreceu a maior retenção de nutrientes, quando comparado com a forma triturada. A ração peletizada contendo o bagaço de mandioca aumentou significativamente o peso da coxa, sobrecoxa e peito.

Houve efeito quadrático do nível de BM sobre o teor de $\mathrm{EE}$ na carne cozida: $\mathrm{EE}=18,90+0,384 \mathrm{NBM}$ $-0,009 \mathrm{NBM}^{2}\left(\mathrm{P}=0,029 \mathrm{R}^{2}=0,71\right)$, onde o nível de $21,33 \%$ de inclusão de BM correspondeu a 19,21\% de deposição de EE na sobrecoxa. Já para o teor de EE na carne crua houve aumento linear: $\mathrm{EE}=19,22$ $+0,135 \mathrm{NBM}\left(\mathrm{P}<0,001 \mathrm{r}^{2}=0,95\right)$.

Esses resultados podem estar relacionados com o tipo de fibra muscular encontrado na sobrecoxa, pois a musculatura da perna dos frangos tem maior quantidade de fibras oxidativas de contração lenta (SO ou tipo I), que se caracteriza por serem pequenas, com maior quantidade de mitocôndrias e mioglobina, o que confere maior coloração vermelha (SATORI et al., 2003; MADEIRA et al., 2006; SIQUEIRA et al., 2007). E ainda de acordo com Lima (2010) na sobrecoxa, comparada com o peito e coxa, há uma maior predisposição para deposição de gordura, pois se caracteriza por ser uma região de reserva energética, servindo também de isolante térmico.

Para o teor de EB na carne cozida encontrou-se redução linear: $\mathrm{EB}=4584-37,20 \mathrm{NBM}(\mathrm{P}=0,003$ $\mathrm{r}^{2}=0,83$ ) e na carne crua não houve efeito do NBM. Como já é sabido no processo de cozimento junto com a saída de água, ocorrem perdas de outros componentes como gordura. Esse fato, aliado a composição do BM em alto teor de energia, advindo do alto teor de fibra e amido, pode ter influenciado nesse resultado.
Também houve redução linear no teor de CZ: $\mathrm{CZ}=4,05-0,018 \mathrm{NBM}\left(\mathrm{P}=0,005 \mathrm{r}^{2}=0,86\right)$. Este resultado está de acordo com aqueles observados na carne da coxa, podendo ser explicado também pelo arraste fecal de nutrientes causado pelo teor e tipo de fibra do BM, possuindo boa parte de fibra solúvel.

Não foi observada influência dos níveis de BM para o teor de MS.

\section{Análises químicas da carne do peito}

Não houve interação significativa $(\mathrm{P}>0,05)$ entre a COC x NBM em nenhuma das variáveis avaliadas. Houve efeito quadrático no teor de MS e $\mathrm{PB}: \mathrm{MS}=29,33-0,33 \mathrm{NBM}+0,007 \mathrm{NBM}^{2}(\mathrm{P}=0,027$; $\mathrm{R}^{2}=0,91$ ), onde o nível de $2,35 \%$ de inclusão de $\mathrm{BM}$ refletiu em 29,56\% de deposição de $\mathrm{MS} ; \mathrm{PB}=87,22$ $+0,35 \mathrm{NBM}-0,013 \mathrm{NBM}^{2}\left(\mathrm{P}=0,056 ; \mathrm{r}^{2}=0,73\right)$, com nível estimado de $13,46 \%$ de inclusão de $\mathrm{BM}$ correspondente a $88,56 \%$ de PB. Já os teores de EE, $\mathrm{CZ}$ e EB não foram influenciados pelos níveis de inclusão de BM ( $\mathrm{P}>0,05)$. (Tabela 7).

Os resultados da $\mathrm{PB}$ da carne do peito são semelhantes aos encontrados na sobrecoxa e também podem ser explicados de acordo com Arruda et al. (2003) incorrendo em maior retenção proteica na carne devido a alta quantidade do amido e solubilidade da fibra do BM, bem como, um melhor aproveitamento da quantidade de proteína contida no ingrediente para atendimento de suas necessidades nutricionais. Já os resultados de deposição de MS assemelham-se aos encontrados na coxa e possivelmente também influenciados pelo arraste de nutrientes causados pela ingestão de fibras do BM. 
Tabela 7. Análises químicas da carne do peito crua e cozida de frangos caipiras alimentados com níveis crescentes de bagaço de mandioca.

\begin{tabular}{|c|c|c|c|c|c|c|c|c|c|c|}
\hline \multirow[t]{2}{*}{ Variável } & \multirow{2}{*}{$\begin{array}{l}\text { Cocção } \\
\text { (COC) }\end{array}$} & \multicolumn{4}{|c|}{$\begin{array}{l}\text { Nível de bagaço de mandioca } \\
(\mathrm{NBM})(\%)\end{array}$} & \multirow[t]{2}{*}{ Geral } & \multirow[t]{2}{*}{$\mathrm{CV}^{1}$} & \multicolumn{3}{|c|}{$\mathrm{P}>\mathrm{F}^{2}$} \\
\hline & & 0 & 10 & 20 & 30 & & & $\mathrm{COC}$ & NBM & $\mathrm{COC} \times \mathrm{NBM}$ \\
\hline \multirow{3}{*}{ MS (\%) } & Sim & 30,95 & 28,70 & 29,60 & 29,90 & 29,86 & \multirow{3}{*}{3,94} & \multirow{3}{*}{$<0,001$} & \multirow{3}{*}{0,007} & \multirow{3}{*}{0,783} \\
\hline & Não & 27,90 & 26,39 & 26,20 & 26,86 & 26,87 & & & & \\
\hline & $\mathrm{Geral}^{3}$ & 29,42 & 27,42 & 27,89 & 28,76 & & & & & \\
\hline \multirow{3}{*}{ PB (\%) } & Sim & 91,02 & 91,82 & 91,89 & 87,66 & 90,59 & \multirow{3}{*}{3,37} & \multirow{3}{*}{$<0,001$} & \multirow{3}{*}{0,028} & \multirow{3}{*}{0,468} \\
\hline & Não & 84,13 & 84,88 & 88,14 & 83,72 & 85,06 & & & & \\
\hline & $\mathrm{Geral}^{3}$ & 87,58 & 88,35 & 90,22 & 85,69 & & & & & \\
\hline \multirow{3}{*}{ EE (\%) } & Sim & 6,65 & 5,73 & 5,86 & 6,76 & 2,85 & \multirow{3}{*}{21,19} & \multirow{3}{*}{$<0,001$} & \multirow{3}{*}{0,101} & \multirow{3}{*}{0,911} \\
\hline & Não & 2,82 & 2,25 & 2,57 & 3,53 & 6,22 & & & & \\
\hline & Geral & 4,74 & 3,99 & 4,62 & 4,74 & & & & & \\
\hline \multirow{3}{*}{ CZ (\%) } & Sim & 4,00 & 4,19 & 4,08 & 3,88 & 4,04 & \multirow{3}{*}{10,31} & \multirow{3}{*}{0,579} & \multirow{3}{*}{0,500} & \multirow{3}{*}{0,597} \\
\hline & Não & 3,94 & 4,30 & 3,98 & 4,25 & 4,12 & & & & \\
\hline & Geral & 3,97 & 4,24 & 4,03 & 4,07 & & & & & \\
\hline \multirow{3}{*}{$\begin{array}{c}\text { EB (kcal/ } \\
\text { kg) }\end{array}$} & Sim & 2581 & 2925 & 2676 & 2510 & 2673 & \multirow{3}{*}{6,43} & \multirow{3}{*}{$<0,001$} & \multirow{3}{*}{0,128} & \multirow{3}{*}{0,412} \\
\hline & Não & 4864 & 4916 & 4945 & 4833 & 4887 & & & & \\
\hline & Geral & 3723 & 3921 & 3684 & 3671 & & & & & \\
\hline
\end{tabular}

${ }^{1}$ Coeficiente de variação (\%); ${ }^{2}$ Significância do teste $\mathrm{F}$ da análise de variância; ${ }^{3}$ Efeito quadrático: $\mathrm{MS}=29,33-0,33 \mathrm{NBM}+0,007 \mathrm{NBM}^{2}$ $\left(\mathrm{P}=0,027 ; \mathrm{R}^{2}=0,91\right), \mathrm{PB}=87,22+0,35 \mathrm{NBM}-0,013 \mathrm{NBM}^{2}\left(\mathrm{P}=0,056 \mathrm{r}^{2}=0,73\right)$.

Fonte: Elaboração dos autores.

\section{Perdas de peso por cozimento}

A inclusão do bagaço de mandioca afetou as perdas de peso por cozimento na carne da coxa (PPCC), ocorrendo efeito quadrático $\left(\mathrm{PPC}=0,031175 \mathrm{BM}^{2}-1,18363 \mathrm{BM}+28,6907, \mathrm{R}^{2}=\right.$ $0,78)$, no qual o nível de $18,63 \%$ de inclusão de bagaço de mandioca refletiu em 17,45\% de perdas de peso por cozimento. Para perdas de peso por cozimento na carne da sobrecoxa (PPCSC) e perdas de peso por cozimento na carne do peito (PPCP), não houve influência do uso do bagaço de mandioca $(\mathrm{P}>0,05)$ (Tabela 8). A perda de água pode variar com a forma em que a mesma se encontra ligada no tecido muscular. Ela pode estar no espaço extracelular, com maior facilidade de ser perdida, ou no interior das miofibrilas, onde para cada unidade de ganho de proteína cerca de três unidades de água são depositadas em associação a esta proteína, sendo esta de difícil remoção (LANNA, 1997; HUFF-LONERGAN; LONERGAN, 2005).

Este fato explicaria o efeito quadrático da inclusão do BM sobre a perda de peso por cozimento da carne da coxa, pois foi observado um aumento da deposição de PB até o nível de 19,53\% de inclusão de bagaço de mandioca na carne crua, assim, uma maior deposição de proteína provocaria a maior agregação das moléculas de água o que implica em menores perdas por cozimento. 
Tabela 8. Análises de Perdas por Cozimento da carne da coxa (PPCC), sobrecoxa (PPCSC) e peito (PPCP) de frangos caipiras alimentados com níveis crescentes de inclusão de bagaço de mandioca.

\begin{tabular}{cccc}
\hline Níveis de inclusão & \multicolumn{3}{c}{ Perdas por Cozimento } \\
\cline { 2 - 4 } Bagaço de Mandioca & PPCC $\%$ & PPCSC \% & PPCP \% \\
\hline $0 \%$ & 29,67 & 26,85 & 17,99 \\
$10 \%$ & 17,01 & 24,08 & 14,01 \\
$20 \%$ & 20,44 & 23,92 & 16,53 \\
$30 \%$ & 20,25 & 21,67 & 14,31 \\
$\mathrm{CV}$ & 21,88 & 23,15 & 32,25 \\
$\mathrm{P}<0,05$ & 0,004 & 0,55 & 0,56 \\
Regressão & $\mathrm{Q}$ & $\mathrm{NS}$ & $\mathrm{NS}$ \\
\hline
\end{tabular}

$\mathrm{PPCC}=$ Perda de peso por cozimento na coxa, $\mathrm{PPCSC}=$ Perda de peso por cozimento na sobrecoxa, $\mathrm{PPCP}=$ Perda de peso por cozimento no peito, $\mathrm{CV}=$ coeficiente de variação $(\%), \mathrm{NS}=$ não significativo a $5 \%$ de probabilidade. $\mathrm{Q}=$ efeito quadrático $(\mathrm{P}=0,0085)$.

Fonte: Elaboração dos autores.

Os resultados encontrados são semelhantes aos de Komiyama et al. (2010) que avaliaram as perdas de peso por cozimento na carne do peito de matrizes de descarte, pelo método de cozimento em chapa metálica, e encontraram a média de perda de peso por cozimento de $17,92 \%$.

\section{Análises de cor, pH e textura}

Os dados do presente trabalho não mostram efeito $(\mathrm{P}>0,05)$ do bagaço de mandioca para os valores de $\mathrm{pH}$, cor e textura na carne do peito, sobrecoxa e coxa (Tabela 9).

A uniformidade da cor e consistência da carne são atributos importantes através dos quais os consumidores irão selecionar produtos de origem animal e finalmente, avaliar a qualidade final do produto (QIAO et al., 2001; QIAO et al., 2002). Como os resultados não foram significativos, a utilização de até 30\% de inclusão de BM não alterou as caracteristicas de cor. Estes resultados contrariam Du e Ahn (2002) que afirmaram que as condições ambientais, tais como alimentos e condições de habitação, podem afetar a cor da carne, o que também não foi observado neste estudo.
Porém, também há outros trabalhos que demonstram que a alimentação tem pouca influência na coloração da carne, sendo mais significativa na cor da pele. A cor da carne sofre influência principalmente pelas condições causadas por metabolismo muscular postmortem acelerado e pensa-se estar relacionado com uma condição hereditária (QIAO et al., 2002; GARCIA et al., 2005; SOUZA et al., 2011).

Qiao et al. (2001) realizaram um estudo com o objetivo de caracterizar a relação da coloração da carne de peito de frangos de corte sobre as propriedades funcionais e físicas da carne. Os filés de peito de frangos foram separados em três grupos de acordo com a luminosidade $\left(\mathrm{L}^{*}\right)$ da carne: carne mais clara que o normal $\left(\mathrm{L}^{*}>53\right)$, carne com cor normal $\left(48<\mathrm{L}^{*}<53\right)$, e carne mais escura do que o normal $\left(\mathrm{L}^{*}<46\right)$. De acordo com esse sistema, as amostras de carne dos cortes do peito, sobrecoxa e coxa do presente trabalho, podem ser classificadas como luminosidade mais clara que o normal.

Porém, a cor ainda sofre influencia do valor do $\mathrm{pH}$, pois quando o $\mathrm{pH}$ da carne está acima do ponto isoelétrico das proteínas miofibrilares, as moléculas de água estão firmemente presas, influenciando em maior absorção de luz pelo músculo, então a carne aparece na cor escura (SALAKOVÁ et al., 2009). 
Tabela 9. Médias de cor ( $\left.\mathrm{L}^{*}, \mathrm{a}^{*}, \mathrm{~b}^{*}\right), \mathrm{pH}$ e força de cisalhamento (FC) da carne do peito, sobrecoxa e coxa de frangos caipiras alimentados com níveis crescentes de inclusão de bagaço de mandioca.

\begin{tabular}{|c|c|c|c|c|c|}
\hline \multicolumn{6}{|c|}{ PEITO } \\
\hline Tratamento & $\mathrm{L}^{*}$ & $a^{*}$ & $\mathrm{~b}^{*}$ & $\mathrm{pH}$ & $\mathrm{FC}(\mathrm{Kgf})$ \\
\hline $0 \%$ & 61,17 & 11,52 & 11,99 & 5,92 & 1,66 \\
\hline $10 \%$ & 61,41 & 9,54 & 12,38 & 5,88 & 1,45 \\
\hline $20 \%$ & 61,23 & 10,87 & 13,06 & 5,87 & 1,40 \\
\hline $30 \%$ & 60,75 & 11,31 & 9,07 & 5,93 & 1,35 \\
\hline $\mathrm{CV}$ & 2,98 & 17,75 & 21,47 & 1,37 & 27,32 \\
\hline $\mathrm{P}<0,05$ & NS & NS & NS & NS & NS \\
\hline \multicolumn{6}{|c|}{ SOBRECOXA } \\
\hline Tratamento & $\mathrm{L}^{*}$ & $a^{*}$ & $\mathrm{~b}^{*}$ & $\mathrm{pH}$ & FC (Kgf) \\
\hline $0 \%$ & 57,52 & 12,17 & 7,99 & 6,43 & 1,19 \\
\hline $10 \%$ & 58,91 & 10,83 & 7,07 & 6,45 & 1,18 \\
\hline $20 \%$ & 58,65 & 10,87 & 7,25 & 6,40 & 1,04 \\
\hline $30 \%$ & 58,29 & 11,94 & 6,57 & 6,48 & 1,37 \\
\hline $\mathrm{CV}$ & 2,89 & 8,90 & 22,44 & 2,72 & 21,94 \\
\hline $\mathrm{P}<0,05$ & NS & NS & NS & NS & NS \\
\hline \multicolumn{6}{|c|}{ COXA } \\
\hline Tratamento & $\mathrm{L}^{*}$ & $a^{*}$ & $\mathrm{~b}^{*}$ & $\mathrm{pH}$ & $\mathrm{FC}(\mathrm{Kgf})$ \\
\hline $0 \%$ & 61,20 & 9,50 & 5,22 & 6,66 & 0,97 \\
\hline $10 \%$ & 60,15 & 10,30 & 7,42 & 6,45 & 1,12 \\
\hline $20 \%$ & 59,92 & 9,40 & 6,59 & 6,43 & 1,09 \\
\hline $30 \%$ & 60,82 & 10,07 & 6,88 & 6,60 & 1,08 \\
\hline $\mathrm{CV}$ & 3,63 & 11,08 & 23,41 & 2,65 & 17,81 \\
\hline $\mathrm{P}<0,05$ & NS & NS & NS & NS & NS \\
\hline
\end{tabular}

$\mathrm{L}^{*}=$ luminosidade, $\mathrm{a}^{*}=$ vermelho, $\mathrm{b}^{*}=$ amarelo, $\mathrm{pH}=$ potencial hidrogeniônico, $\mathrm{FC}=$ força de cisalhamento em quilograma força (kgf), $\mathrm{CV}=$ coeficiente de variação (\%), $\mathrm{NS}=$ não significativo a $5 \%$ de probabilidade.

Fonte: Elaboração dos autores.

Além disso, um baixo $\mathrm{pH}$ final reduz a importância da mioglobina em seletivamente absorver a luz verde, resultando em carne que parece menos vermelho $\left(\mathrm{a}^{*}\right)$ e mais amarelo $\left(\mathrm{b}^{*}\right)$ (CASTELLINI; MUNGNAI; BOSCO, 2002). No entanto, observa-se no presente trabalho que os valores de $a^{*}$ são maiores que os valores $b^{*}$ nos três cortes estudados. E considerando o trabalho de Qiao et al. (2001), no qual a carne com coloração mais escura, carne com coloração normal e, carne de cor clara, correspondem, respectivamente a $\mathrm{pH}$ de 6,$23 ; 5,96$; e 5,81 , o que classifica o corte da coxa e sobrecoxa dentro da faixa de coloração mais escura por apresentarem maior valor de $\mathrm{pH}$ quando comparado com o corte do peito.

De acordo com Ramos e Gomide (2007) a textura é classificada da seguinte forma: valores abaixo de 3,62 kgf carne extremamente macia; entre 6,62 a 9,60 kgf são ligeiramente macio a ligeiramente duro; e acima de 12,60 kgf extremamente duro. Estes são valores que estão muito acima dos observados no presente estudo (0,97 - 1,66 kgf), no qual podemos considerar a textura da carne dos três cortes (coxa, sobrecoxa e peito) como extremamente macia. Ou ainda de acordo com Ali et al. (2007) a força de cisalhamento decresce com o tempo de armazenamento, o que pode ter influenciado no presente estudo, pois as aves ficaram armazendas pelo período de 6 meses em freezer até a realização das análises.

Segundo Kerry, Kerry e Ledward (2002) as mudanças organolépticas podem ser fortemente 
influênciadas pela alimentação, pois ao aumentar a energia da ração os animais conseguem acumular maior quantidade de gordura, o que torna a carne mais macia e suculenta, no entanto, para que esta mudança ocorra é necessário uma mudança brusca na alimentação o que não ocorreu, pois as rações foram formuladas com níveis isonutrientes para proteína e energia.

Os resultados encontrados para textura da carne de frango tipo caipira alimentado com níveis crescentes de BM são atribuídos à homogeneidade de condições com que foi realizado o processamento no momento do abate, já que este é um fator que potencialmente influencia as características instrumentais da carne (VENTURINI; SARCINELLI; SILVA, 2007).

Conforme observado no presente estudo, a utilização de BM não afetou as características instrumentais da carne. Em função disso, a opção pela utilização de até $30 \%$ de inclusão do BM em inclusão na dieta de frangos tipo caipiras pode ser definida pelo preço e disponibilidade no mercado.

\section{Conclusão}

A inclusão do bagaço de mandioca na dieta e a cocção afetaram as características química da carne de frangos de corte tipo caipira. Os resultados apontaram que houve redução linear das cinzas na carne da coxa e sobrecoxa, aumento na deposição de proteína na carne da coxa, sobrecoxa e peito e houve aumento na deposição de extrato etéreo somente na carne da sobrecoxa. Contudo as características físicas da carne não recebeu influência pelos níveis de inclusão do BM. Recomenda-se, com base na composição química da carne, utilizar até o nível de $20 \%$ de inclusão do bagaço de mandioca.

\section{Agradecimentos}

Os autores agradecem a CAPES e ao CNPq.

\section{Referências}

ADEYEMI, O. A.; ERUVBETINE, D.; OGUNTONA, T.; DIPEOLU, M.; AGUNBIADE, J. A. Feeding broiler chicken with diets containing whole cassava root meal fermented with rumen filtrate. Archivos de Zootecnia, Córdoba, v. 57, n. 218, p. 247-258, 2008.

ALI, M. S.; KANG, G. H.; YANG, H. S.; JEONG, J. Y.; HWANG, Y. H.; PARK, G. B.; JOO, S. T. A. comparison of meat characteristics between duck and chicken breast. Asian-Australian Journal Animal Science, Seoul, v. 20, n. 6, p. 1002-1006, 2007.

ARRUDA, A. M. V.; LOPES, D. C.; FERREIRA, W. M.; ROSTAGNO, H. S.; QUEIROZ, A. C.; PEREIRA, E. S.; FERREIRA, A. S.; SILVA, J. F. Desempenho e características de carcaça de coelhos alimentados com rações contendo diferentes níveis de amido e fontes de fibra. Revista Brasileira de Zootecnia, Viçosa, MG, v. 32, n. 6, p. 1311-1320, 2003.

BRASIL, MAPA. Oficio Circular DOI/DIPOA No 007 de 19 de maio 1999. Dispõe sobre as normas para criação de frango caipira e produção de ovos caipiras. Brasília, DF, 1999. Disponível em: <http://www.agricultura.gov. br/sislgis $>$. Acesso em: 04 ago 2011.

CASTEllini, C.; MUNGNAI, C.; BOsCO, A. D. A Effect of organic production system on broiler carcass and meat quality. Meat Science, Amsterdam, v. 60, n. 3, p. 219-225, 2002.

DU, M.; AHN, D. Effect of dietary conjugated linoletic acid on the growth rate of live birds and on the abdominal fat content and quality of broiler meat. Poultry Science, Savoy, v. 81, n. 3, p. 428-433, 2002.

FENIMAN, C. M. Caracterização de raizes de mandioca (Manihot esculenta Crantz) da cultivar IAC 576-70 quanto à cocção, composição química e propriedades do amido em duas épocas de colheita. 2004. Dissertação (Mestrado em Ciência e Tecnologia de Alimentos) - Escola Superior de Agricultura Luiz de Queiroz. Universidade de São Paulo, Piracicaba.

GARCIA, R. G.; MENDES, A. A.; COSTA, C.; PAZ, I. C. L. A.; TAKAHASHI, S. E.; PELÍCIA, K. P.; KOMIYAMA, C. M.; QUINTEIRO, R. R. Desempenho e qualidade da carne de frangos de corte alimentados com diferentes níveis de sorgo em substituição ao milho. Arquivo Brasileiro de Medicina Veterinária e Zootecnia, Belo Horizonte, v. 57, n. 5, p. 634-643, 2005.

HUFF-LONERGAN, E.; LONERGAN, S. M. Mechanism sofwater-holding capacity of meat: the role of postmortem biochemical and structural changes. Meat Science, Amsterdam, v. 71, p. 194-204, 2005. 
KERRY, J.; KERRY, J.; LEDWARD, D. Meat processing improving quality. Londres: CRC, 2002. $464 \mathrm{p}$.

KOMIYAMA, C. M.; MENDES, A. A.; SANFELICE, C.; CANIZARES, M. C.; ROÇA, R. O.; TAKAHASHI, S. E.; RODRIGUES, L.; CANIZARES, R. O.; PAZ, I. C. L. A.; CARDOSO, K. F. G. Qualidade físico-química e sensorial da carne de peito de matrizes pesadas de descarte. Ciência Rural, Santa Maria, v. 40, n. 7, p. 16231629, 2010.

LANNA, D. P. Fatores condicionantes e predisponentes da puberdade e da idade de abate. In: PEIXOTO, A. M.; MOURA, J. C.; FARIA, V. P. (Coord.). Produção do novilho de corte. Piracicaba: FEALQ, 1997. p. 41-78.

LIMA, M. R. A carne de frango e suas características. Santa Catarina: Agromais, 2010, Disponível em: <http:// pt.engormix.com/MAavicultura/administracao/artigos/ carne-frango-caracteristicas-t272/p0.htm $>$. Acesso em: 02 maio 2013.

MADEIRA, L. A.; SATORI, J. R.; SALDANHA, E. S. P. B.; PIZZOLANTE, C. C.; SILVA, M. D. P.; MENDES, A. A.; TAKAHASHI, S. E.; SOLARTE, W. V. N. Morfologia das fibras musculares e esqueléticas de frangos de corte de diferentes linhagens criados em sistema de confinamento e semiconfinamento. Revista Brasileira de Zootecnia, Viçosa, MG, v. 35, n. 6, p. 23222332, 2006.

MARQUES, J. A.; PRADO, I. N.; ZEOULA, L. M.; ALALDE, C. R.; NASCIMENTO, W. G. do. Avaliação da mandioca e seus resíduos industriais em substituição ao milho no desempenho de novilhas confinadas. Revista Brasileira de Zootecnia, Viçosa, MG, v. 29, n. 5, p. 15281536, 2000.

QIAO, M.; FLETCHER, D. L.; NORTHCUTT, J. K.; SMITH, D. P. The relationship between rawbroiler breast meat colour and composition. Poultry Science, Savoy, v. 81, p. 422-427, 2002.

QIAO, M.; FLETCHER, D. L.; SMITH, D. P.; NORTHCUTT, J. K. The effect of broiler breast meat color on $\mathrm{pH}$, moisture, water holding capacity, and emulsification capacity. Poultry Science, Savoy, v. 80, p. 676-680, 2001.

RAMOS, E. M.; GOMIDE, L. A. M. Avaliação da qualidade de carnes: fundamentos e metodologias. Viçosa, MG: Ed. UFV, 2007. 599 p.

RAUPP, D. da S.; MARQUES, S. H. de P.; ROSA, D. A.; CALDI, C. M.; CREMASCO, A. C. V.; BANZATTO, D. A. Arraste via fecal de nutrientes da ingestão produzido por bagaço de mandioca hidrolisado. Scientia Agrícola, Piracicaba, v. 59, n. 2, p. 235-242, 2002.
ROSTAGNO, H. S.; ALBINO, L. F. T.; DONZELE, J. L.; GOMES, P. C.; OLIVEIRA, R. F.; LOPES, D. C.; FERREIRA, A. S.; BARRETO, S. L. T.; EUCLIDES, R. F. Tabela brasileira para aves e suínos: composição de alimentos e exigências nutricionais. 3. ed. Viçosa, MG: UFV, DZO, 2011. 252 p.

ROSTAGNO, H. S.; ALBINO, L. F. T.; DONZELE, J. L.; GOMES, P. C.; OLIVEIRA, R. F.; LOPES, D. C.; FERREIRA, A. S.; BARRETO, S. L. T. Tabela brasileira para aves e suínos: composição de alimentos e exigências nutricionais. 3. ed. Viçosa, MG: UFV/DZO, 2005. 186 p.

SALAKOVÁ, A.; STRAKOVÁ, E.; VALKOVÁ, V.; BUCHTOVÁ, H.; STEINHAUSEROVÁ, I. Quality of chicken broiler raw and cooked meat depending on their sex. Acta Veterinária, Mossoró, v. 78, n. 3, p. 497-504, 2009.

SATORI, J. R.; GONZALES, E.; MACARI, M.; PAI, V. D.; OLIVEIRA, H. N. de. Tipos de fibras no músculo fleoxor longo do hálux de frangos de corte submetidos ao estresse pelo calor e frio e alimentos em "pair-feeding". Revista Brasileira de Zootecnia, Viçosa, MG, v. 32, n. 4, p. 918-925, 2003.

SILVA, D. J.; QUEIROZ, A. C. Análise de alimentos, métodos químicos e biológicos. 3. ed. Viçosa: UFV, 2006. 235 p.

SIQUEIRA, J. C.; OLIVEIRA, R. F. M.; DONZELE, J. L.; CECON, P. R.; BALBINO, E. M.; OLIVEIRA, W. P. Níveis de lisina digestível da ração e temperatura ambiente para frangos de corte em crescimento. Revista Brasileira de Zootecnia, Viçosa, MG, v. 36, n. 6, p. 20542062, 2007.

SOUSA, J. P. L.; RODRIGUES, K. F.; ALBINO, L. F. T.; SANTOS NETA, E. R.; VAZ, R. G. M. V.; PARENTE, I. P.; SILVA, G. F.; AMORIM, A. F. Bagaço de mandioca em dietas de frango de corte. Revista brasileira de saúde e produção animal, Salvador, v. 13, n. 4, p. 1044-1053, 2012.

SOUZA, K. M. R.; CARRIJO, A. S.; KIEFER, C.; FASCINA, V. B.; FALCO, A. L.; MANVAILER, G. V.; GARCÍA, A. M. L. Farelo da raiz integral de mandioca em dietas de frangos de corte tipo caipira. Archivos de Zootecnia, Córdoba, v. 60, n. 231, p. 489-499, 2011.

STATISTICAL ANALYSES SYSTEM - SAS. SAS/ INSIGHT User'sguide. Versão 9.0 - versão para Windows. Cary: SAS Institute, 2002. CD-ROM.

UNIÃO BRASILEIRA DE AVICULTURA - UBABEF. Relatório anual 2011/2012. Porto Alegre: Asgav, 2012. Disponível em: <http://www.abef.com.br/ubabef/ exibenoticiaubabef.php?notcodigo $=2761>$. Acesso em: 26 fev. 2013. 
VENTURINI, K. S.; SARCINELli, M. F.; SILVA, L. C. ZANUSSO, J. T.; DIONELlO, N. J. L. Produção Características da carne de frango. Espírito Santo: PIE- avícola alternativa - análise dos fatores qualitativos da UFES, 2007. 7 p. (Boletim técnico, n. 1307). carne de frangos de corte tipo caipira. Revista Brasileira Agrociência, Pelotas, v. 9, n. 3, p. 191-194, 2003. 